\title{
Acacetin Alleviates Inflammation and Matrix Degradation in Nucleus Pulposus Cells and Ameliorates Intervertebral Disc Degeneration in vivo
}

This article was published in the following Dove Press journal:

Drug Design, Development and Therapy

\author{
Hao Wang ${ }^{1} *$ \\ Zengxin Jiang ${ }^{1} * *$ \\ Zhiying Pang' \\ Tianyao Zhou' \\ Yutong $\mathrm{Gu}^{1,2}$ \\ 'Department of Orthopaedic Surgery, \\ Zhongshan Hospital Affiliated to Fudan \\ University, Shanghai 200032, People's \\ Republic of China; ${ }^{2}$ Department of \\ Orthopaedic Surgery, Shanghai Public \\ Health Clinical Center, Fudan University, \\ Shanghai 201508, People's Republic of \\ China
}

*These authors contributed equally to this work

\begin{abstract}
Purpose: Intervertebral disc degeneration (IDD) is one of the most prevalent musculoskeletal disorders. The nucleus pulposus is the major component of the intervertebral disc, and nucleus pulposus cells (NPCs) play a significant role in the normal functioning of the intervertebral disc. Reactive oxygen species (ROS) generation, inflammation and extracellular matrix degradation in NPCs contribute to the degeneration of intervertebral discs. Acacetin is a drug that exerts antioxidant and anti-inflammatory effects on many types of cells. However, whether acacetin can relieve the degeneration of NPCs remains unknown.
\end{abstract}

Methods: NPCs were extracted from rat intervertebral discs. The NPCs were treated with tert-butyl peroxide (TBHP) to simulate a high-ROS environment, and acacetin was subsequently added. The contents of ROS, inflammatory mediators (COX-2, iNOS) and extracellular matrix components (aggrecan, collagen II, MMP13, MMP9, MMP3) were measured. Components of related signaling pathways (Nrf2, MAPK) were also evaluated. To determine the effect of acacetin in vivo, we simulated disc degeneration via needle puncture. Acacetin was then applied intraperitoneally, and the degenerative status was evaluated using MRI and histopathological analysis.

Results: In vitro, acacetin alleviated TBHP-induced ROS generation and upregulated the expression of antioxidant proteins, including HO-1, NQO1, and SOD. In addition, acacetin relieved the TBHP-induced generation of inflammatory mediators (COX-2, iNOS) and degradation of the extracellular matrix (aggrecan, collagen II, MMP13, MMP9, and MMP3). Acacetin exerted its effect by activating the Nrf2 pathway and inhibiting p38, JNK and ERK1/2 phosphorylation. In vivo, acacetin ameliorated puncture-induced disc degeneration in a rat tail model, which was evaluated using MRI and histopathological analysis.

Conclusion: Acacetin alleviated IDD in vitro and in vivo and may have the potential to be developed as an effective treatment for IDD.

Keywords: acacetin, intervertebral disc degeneration, IDD, reactive oxygen species, ROS, inflammation

\section{Introduction}

Intervertebral disc degeneration (IDD) is the most prevalent musculoskeletal disorder and accounts for nearly $40 \%$ of low back pain etiologies. ${ }^{1,2}$ Degenerative processes lead to morphological and physiological changes in the discs, ultimately 
decreasing the capability for movement and weight bearing. ${ }^{3}$ Current therapies for IDD mainly focus on relieving its symptoms rather than slowing or even reversing the degenerative process. For end-stage symptomatic patients, the surgical removal of the disc and fusion of the adjacent vertebra are inevitable. ${ }^{4}$

Nucleus pulposus cells (NPCs) and their extracellular matrix (ECM) are the main components of the nucleus pulposus. Their proper functioning maintains the structural stability and normal functioning of the intervertebral disc. The mechanism underlying IDD has not been completely elucidated. However, it is generally accepted that ECM degradation and the generation of inflammatory mediators are typical characteristics of the degenerative process. ${ }^{5}$ These inflammatory mediators mainly consist of interleukin-1 $\beta$ (IL-1 $\beta$ ), tumor necrosis factor- $\alpha$ (TNF- $\alpha$ ), nitric oxide (NO) and prostaglandin E2 (PGE2), which inhibit the synthesis of matrix components and facilitate the production of ECM-related catabolic enzymes. ${ }^{6-8}$ Numerous attempts to halt the degeneration process and restore normal functioning through methods such as stem cell transplantation, ${ }^{9}$ anti-inflammatory therapy ${ }^{10}$ and antiangiogenic therapy have shown favorable outcomes. ${ }^{11}$

Recently, accumulating evidence has indicated that the upregulated generation of reactive oxygen species (ROS) plays a crucial role in the occurrence and progression of IDD. ${ }^{12}$ ROS are a series of active oxygen-containing chemical species, including hydrogen peroxide $\left(\mathrm{H}_{2} \mathrm{O}_{2}\right)$, superoxide anion $\left(\mathrm{O}^{2-}\right)$ and free radicals. ${ }^{13}$ Excessive ROS levels have been widely detected in degenerative intervertebral discs. ROS accumulation inside NPCs interferes with normal physiological processes, activating various signaling pathways, including the nuclear factor kappa-B (NF- $\mathrm{kB})$ and mitogenactivated protein kinase (MAPK) pathways, ${ }^{14,15}$ which then promote the expression of inflammatory mediators and ultimately accelerate ECM degradation. Scavenging intracellular ROS may help to alleviate the degeneration process. Multiple categories of drugs have been proven to be capable of scavenging ROS, including nonenzymatic antioxidants, polyphenols, ROS scavengers, herbal components and growth factors. ${ }^{16}$ These drugs can either scavenge ROS directly or work by stimulating the synthesis of endogenous antioxidant proteins.

Acacetin (5,7-dihydroxy-4'-methoxyflavone) is one of the main compounds isolated from Agastache rugosa, a common traditional Chinese herb. Existing studies have shown that acacetin has antidiabetic, ${ }^{17}$ antidepressant, ${ }^{18}$ anticancer, ${ }^{19}$ antioxidant ${ }^{20}$ and anti-inflammatory ${ }^{21}$ properties. However, there has been no research concerning the therapeutic effect of acacetin on IDD. Thus, this study focused on the ROSscavenging function of acacetin in rat NPCs and the underlying mechanisms. Moreover, rats with coccygeal intervertebral disc puncture-induced IDD were intraperitoneally injected with acacetin to determine the protective function of acacetin in vivo.

\section{Materials and Methods Reagents and Antibodies}

Acacetin was obtained from Selleck Chemicals (Houston, Texas, United States) and diluted in dimethyl sulfoxide (DMSO, Sigma-Aldrich, St. Louis, MO, United States). Cell Counting Kit-8, HRP-labeled goat anti-rabbit IgG, the Nuclear Protein and Cytoplasmic Protein Extraction Kit, electrochemiluminescence reagent, the Reactive Oxygen Species Assay Kit and the SOD Assay Kit with WST-8 were purchased from Beyotime (Shanghai, China). Tertbutyl peroxide (TBHP) was purchased from Sigma-Aldrich (St. Louis, MO, United States). Primary antibodies against aggrecan, collagen II, MMP3 and MMP13 were obtained from Abcam (Cambridge, MA, United States). Antibodies against MMP9, HO-1, NQO1, Nrf2, Lamin B1, and GAPDH were purchased from the Proteintech Group (Wuhan, China). Antibodies against p38, p-p38, extracellular signal-regulated kinase (ERK)1/2, p-ERK1/2, c-Jun N-terminal kinase (JNK), and p-JNK were obtained from Cell Signaling Technology (Danvers, MA, United States). Collagenase type II, DMEM/ F12 culture medium, fetal bovine serum, penicillin and streptomycin, and a $0.25 \%$ trypsin EDTA solution were purchased from Gibco (Grand Island, NY, United States). Reverse Transcript Master Mix and qPCR Master Mix were purchased from Promega (Madison, WI, United States). TRIzol reagent was obtained from Invitrogen (Carlsbad, CA, United States). Polyvinylidene difluoride (PVDF) membranes were purchased from Millipore (Bedford, MA, United States).

\section{Experimental Animals}

For primary cell isolation and the generation of an IDD rat model, four- and eight-week-old male Sprague-Dawley (SD) rats were obtained from the Shanghai SLAC Laboratory Animal Company (Shanghai, China). The protocols for the animal care and use of the laboratory animals were approved by the Ethics Committee of Zhongshan Hospital. The operations were conducted in accordance with the National Institutes of Health (NIH) Guide for the Care and Use of Laboratory Animals. 


\section{Primary Nucleus Pulposus Cell Isolation and Culture}

Primary NPCs were isolated from SD rats. Four-week-old SD rats were euthanized by injecting an excess amount of sodium pentobarbital. Death was confirmed by checking their breathing and heartbeat. The verification of death was supplemented by percutaneous cardiac puncture. The gellike tissue was separated from the lumbar discs and digested with $0.1 \%$ collagenase type II for two hours at $37^{\circ} \mathrm{C}$. The isolated NPCs were centrifuged, resuspended and cultured in DMEM/F12 culture medium with $10 \%$ fetal bovine serum, $100 \mathrm{U} / \mathrm{mL}$ penicillin and $100 \mu \mathrm{g} / \mathrm{mL}$ streptomycin. Upon reaching $80 \%-90 \%$ confluency, the NPCs were passaged using a $0.25 \%$ trypsin EDTA solution. The culture medium was replaced every two days, and NPCs between passage one and passage three were used in the experiments.

\section{Cell Viability Assay}

The viability of NPCs was measured using the Cell Counting Kit-8 (CCK-8). The NPCs were cultured in 96well plates at a density of 5000 cells per well for 24 hours. The cells were then treated with different concentrations of acacetin $(0 \mu \mathrm{M}, 0.1 \mu \mathrm{M}, 0.3 \mu \mathrm{M}, 1 \mu \mathrm{M}, 3$ $\mu \mathrm{M}, 10 \mu \mathrm{M})$ or DMSO for 24 or 48 hours. After the intervention, the culture medium was removed, and the NPCs were washed with phosphate-buffered saline (PBS). One hundred microliters of serum-free DMEM/ F12 solution containing $10 \mu \mathrm{L}$ of CCK-8 was added to each well. The plates were then incubated at $37^{\circ} \mathrm{C}$ for two hours, after which the absorbance at $450 \mathrm{~nm}$ was measured using a spectrophotometer (FlexStation 3, Molecular Devices, San Jose, CA, United States).

\section{Western Blotting}

Total protein was extracted from the NPCs using RIPA lysis buffer with a protease and phosphate inhibitor cocktail. Nuclear protein was extracted using the Nuclear and Cytoplasmic Protein Extraction Kit according to the manufacturer's instructions. Protein was separated through sodium dodecyl sulfate-polyacrylamide gel electrophoresis (SDSPAGE) and transferred to polyvinylidene difluoride (PVDF) membranes. After blocking with a 5\% nonfat milk solution, the membranes were incubated with the primary antibodies overnight. The membranes were then washed with TBST three times and incubated with the respective secondary antibodies for one hour. Finally, the blots were visualized via enhanced chemiluminescence on an imaging system (Tanon, Shanghai, China).

\section{Real-Time PCR}

The total RNA of the NPCs was extracted and isolated using TRIzol reagent according to the manufacturer's instructions. The concentration and quality of the extracted RNA were measured on a Nanodrop spectrophotometer (DeNovix, Wilmington, DE, United States), and the cDNA was synthesized from $1 \mu \mathrm{g}$ of the RNA using Reverse Transcript Master Mix. qPCR amplification was performed using an Applied Biosystems QuantStudio 5 Real-Time PCR System (Applied Biosystems, Foster City, CA, United States) with a qPCR Master Mix Kit. GAPDH expression was used to normalize the $\mathrm{Ct}$ values. The primers for the genes evaluated in this study are listed in Table 1.

\section{Flow Cytometry}

Flow cytometry was employed to determine the intracellular ROS content using the Reactive Oxygen Species Assay Kit. After the respective treatments, DCFH-DA was added to each

Table I Primers' Sequences Used in the Real-Time PCR

\begin{tabular}{|l|l|l|}
\hline Name & Forward & Reverse \\
\hline Collagen II & ACGCTCAAGTCGCTGAACAACC & ATCCAGTAGTCTCCGCTCTTCCAC \\
Aggrecan & CTTCCCAACTATCCAGCCAT & TCACACCGATAGATCCCAGA \\
MMPI3 & AACCAAGATGTGGAGTGCCTGATG & CACATCAGACCAGACCTTGAAGGC \\
MMP9 & AGGTGCCTCGGATGGTTATCG & TGCTTGCCCAGGAAGACGAA \\
MMP3 & GCTCATCCTACCCATTGCAT & GCTTCCCTGTCATCTTCAGC \\
HO-I & ATACCCGCTACCTGGGTGAC & TGTCACCCTGTGCTTGACCT \\
NQOI & ATCACCAGGTCTGCAGCTTC & GCCATGAAGGAGGCTGCTGT \\
COX-2 & CAAATCCTTGCTGTTCCCACCCAT & GTGCACTGTGTTTGGAGTGGGTTT \\
iNOS & GCTCGGGCTGAAGTGGTATGC & GAAGTCTCGGACTCCAATCTCGGT \\
GAPDH & GACAATTTTGGCATCGTGGA & ATGCAGGGATGATGTTCTGG \\
\hline
\end{tabular}


well at a concentration of $10 \mu \mathrm{M}$, followed by incubation at $37^{\circ} \mathrm{C}$ for 20 minutes. The NPCs were digested from the culture plate and then centrifuged and resuspended. Flow cytometry was used to determine ROS levels at an excitation wavelength of $488 \mathrm{~nm}$ and an emission wavelength of $525 \mathrm{~nm}$.

\section{Superoxide Dismutase (SOD) Activity Measurement}

The total SOD activity of the NPCs was measured using the SOD Assay Kit with WST-8. The NPCs were collected and homogenized. After centrifugation, the supernatant was added to a 96-well plate and mixed with the buffer solution and working solution for 30 minutes. The absorbance at 450 $\mathrm{nm}$ was measured, and SOD activity was calculated according to the formula provided by the manufacturer.

\section{Surgical Procedures}

Eighteen eight-week-old male SD rats were randomly divided into three equal groups: a control group, an IDD group and an acacetin group. The rats in the IDD group and the acacetin group were anesthetized using sodium pentobarbital. Two continuous segments near the tail root were selected to establish the disc degeneration model. The coccygeal intervertebral discs were punctured using a 21-gauge needle at a depth of $5 \mathrm{~mm}$, after which the needle was rotated $360^{\circ}$ and held in place for 30 seconds. ${ }^{22}$ The rats in the acacetin group received an intraperitoneal injection of acacetin at a dose of $25 \mathrm{mg} /$ $\mathrm{kg}$ once a week, and the rats in the control and IDD groups received an injection of an equal volume of saline.

\section{Magnetic Resonance Imaging (MRI)}

Magnetic resonance imaging can detect structural and water content changes in soft tissues. We used T2-weighted imaging (T2WI) to acquire coronal images of rats and evaluated their coccygeal intervertebral discs. The rats received an MRI scan on the same day as surgery and four weeks after surgery. A 3.0T MR scanning system (Siemens 3T Skyra Scanner) was used to scan the rat tails using the following settings: repetition time (TR) $4000 \mathrm{~ms}$, echo time (TE) 76 $\mathrm{ms}$, and slice thickness $1.5 \mathrm{~mm}$.

\section{Histopathological Analysis}

After four weeks, all rats were euthanized by injecting excess sodium pentobarbital. Their tails were collected, fixed in $4 \%$ paraformaldehyde for 24 hours and then decalcified for 48 hours using a rapid decalcification solution composed of formic acid, formaldehyde and hydrochloric acid. The tails were cut in the middle of the vertebrae to obtain intact intervertebral discs. The tissues were then dehydrated, embedded in paraffin and cut into $3-\mu \mathrm{m}$ slices. The slices were stained using hematoxylin-eosin (H\&E), alcian blue $(\mathrm{AB})$ and safranin $\mathrm{O} /$ fast green, after which they were evaluated using a histological score. ${ }^{23}$

\section{Statistical Analysis}

All the experiments were carried out with at least three independent repeats. All data are represented as the mean \pm SD (standard deviation). Statistical analyses were performed using GraphPad Prism 8 (GraphPad Software, Inc., La Jolla, CA, USA). The significance between multiple groups was analyzed using one-way analysis of variance (ANOVA) with a post hoc Tukey HSD test for multiple comparisons. A P-value $<0.05$ was considered statistically significant.

\section{Results}

\section{Effects of Acacetin on the Cell Viability of NPCs}

Figure 1A shows the molecular structure of acacetin. To determine the appropriate concentration for the subsequent experiments, we used the CCK- 8 assay to determine the effects of acacetin on cell viability. As shown in Figure 1B and C, NPCs were treated with different concentrations of acacetin for 24 or 48 hours. A concentration higher than $1 \mu \mathrm{M}$ showed obvious cell toxicity, whereas no significant difference in cell viability was found among the groups treated with concentrations lower than $1 \mu \mathrm{M}$. Thus, we chose concentrations of $0.3 \mu \mathrm{M}$ and $1 \mu \mathrm{M}$ for use in the subsequent experiments.

\section{Acacetin Suppressed the THBP-Induced Generation of ROS}

To ascertain the effect of acacetin on ROS generation in NPCs, we measured intracellular ROS levels after treatment with TBHP $(50 \mu \mathrm{M})$ in the presence or absence of acacetin $(0.3 \mu \mathrm{M}, 1 \mu \mathrm{M})$ using DCFH-DA with flow cytometry. As shown in Figure 2A and B, after 24 hours of intervention, TBHP dramatically increased the intracellular ROS level, while acacetin inhibited TBHP-induced ROS generation in a dose-dependent manner.

\section{Acacetin Upregulated the Expression of Antioxidant Proteins}

ROS-scavenging is carried out by antioxidant proteins. At least 710 kinds of antioxidant proteins that have been 
A

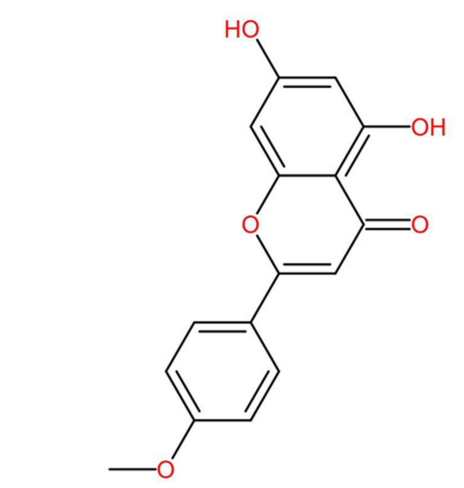

5,7-dihydroxy-2-(4-methoxyphenyl)chromen-4-one
B

24h

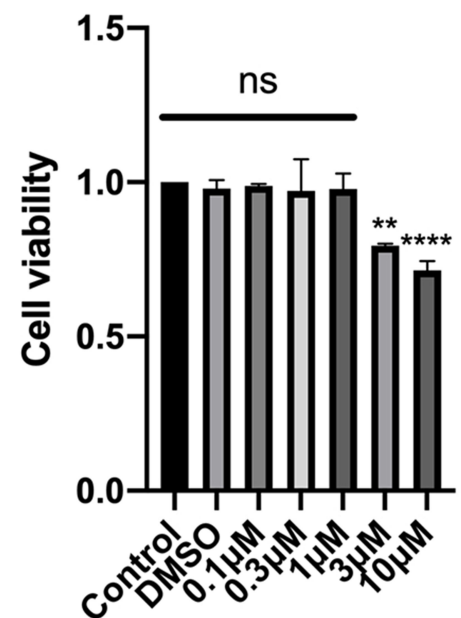

C

48h

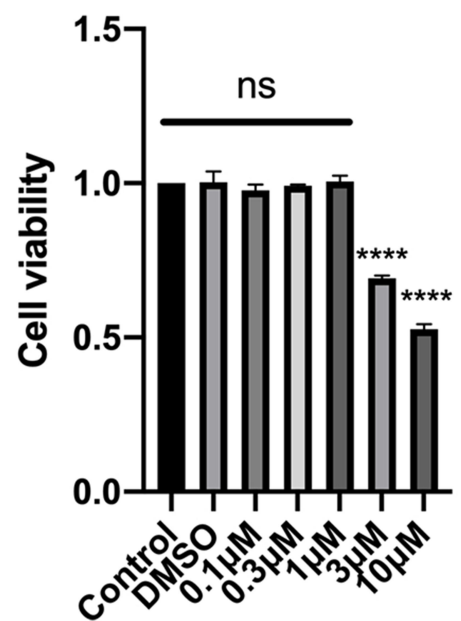

Figure I The chemical structure of acacetin and the effect of acacetin on the viability of the NPCs. (A) The molecular structure of acacetin. (B, C) The NPCs were treated with different concentrations of acacetin for $24(\mathbf{B})$ or $48(\mathbf{C})$ hours, after which the cell viability was measured using a CCK-8 assay. The data are represented as the mean \pm SD. $n=3$. ns: not significant. ${ }^{* *} \mathrm{p}<0.01$, $* * * * \mathrm{p}<0.000$ I versus control.

discovered to date. ${ }^{24} \mathrm{Wu}$ et al found that acacetin is an activator of the nuclear factor erythroid 2-related factor 2 (Nrf2) pathway, ${ }^{25}$ and we thus evaluated the expression of downstream proteins of this pathway, including heme oxygenase-1 (HO-1), NAD(P)H dehydrogenase, quinone 1 (NQO1) and SOD. NPCs were treated with THBP (50 $\mu \mathrm{M})$ in the presence or absence of acacetin $(0.3 \mu \mathrm{M}, 1$ $\mu \mathrm{M}$ ) for 24 hours. As shown in Figure 2C-E, acacetin increased the expression of $\mathrm{HO}-1$ and NQO1 at the mRNA and protein levels in a dose-dependent manner. We then measured the total SOD activity of the NPCs. Figure $2 \mathrm{~F}$ shows that after 24 hours of treatment, acacetin also upregulated SOD activity in a dose-dependent manner.

\section{Acacetin Ameliorated Inflammation and ECM Degradation Caused by TBHP}

Proinflammatory mediators facilitate IDD. NO and PGE2 are important inflammatory mediators, and their synthesis depends on inducible nitric oxide synthase (iNOS) and cyclooxygenase 2 (COX-2), respectively. ${ }^{26}$ NPCs were also treated with TBHP either alone or in combination with acacetin for 24 hours. As shown in Figure 3A-C, TBHP treatment promoted the expression of iNOS and COX-2 at the mRNA and protein levels, while acacetin downregulated their expression levels in a dose-dependent manner.

ECM degradation of NPCs is the main manifestation of IDD. Aggrecan and collagen II are the main ECM components. Figure 3D-I show that the mRNA and protein expression of aggrecan and collagen II was suppressed by TBHP and that acacetin relieved this suppression in a dose-dependent manner. ECM degradation is mediated by matrix metalloproteinases (MMPs). We evaluated the expression levels of MMP13, MMP9 and MMP3 and found that they were upregulated by TBHP and inhibited by acacetin.

\section{Acacetin Activated the Nrf2 Pathway and Inhibited the TBHP-Induced Activation of the MAPK Pathway}

The Nrf2 pathway is closely related to ROS scavenging in cells. Acacetin has been proven to protect cardiomyocytes and lung tissue from oxidative stress through the activation of the Nrf2 pathway. $^{25,27}$ Therefore, we studied whether acacetin activated the Nrf2 pathway in NPCs. NPCs were preincubated with acacetin $(0.3 \mu \mathrm{M}, 1 \mu \mathrm{M})$ for two hours and then treated with TBHP $(50 \mu \mathrm{M})$ for another 24 hours. Nuclear protein was extracted and then evaluated using Western blotting. Figure 4A shows that the intranuclear Nrf2 protein level increased in the preincubation groups, indicating that acacetin could also activate the Nrf2 pathway in NPCs.

Recent studies have demonstrated that the MAPK pathway is closely involved in the degeneration process. ${ }^{28}$ Thus, in this research, we evaluated the activation status of the p38, JNK and ERK pathways. NPCs were also 
A

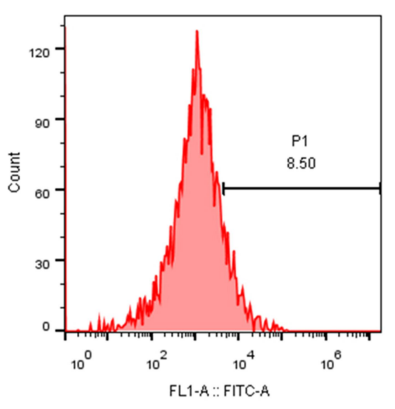

Control

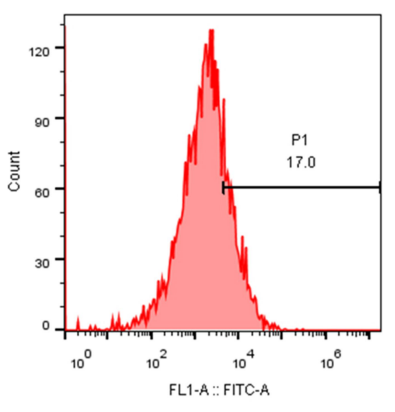

TBHP

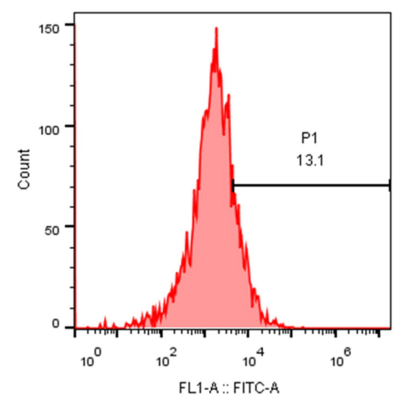

$\mathrm{TBHP}+0.3 \mu \mathrm{M}$ Acacetin

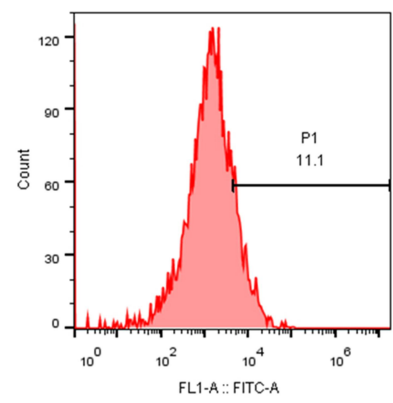

$\mathrm{TBHP}+1 \mu \mathrm{M}$ Acacetin
B

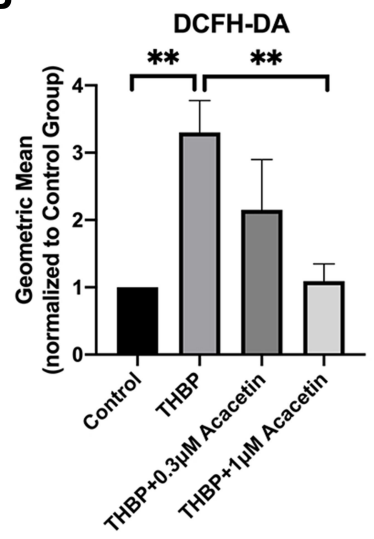

C

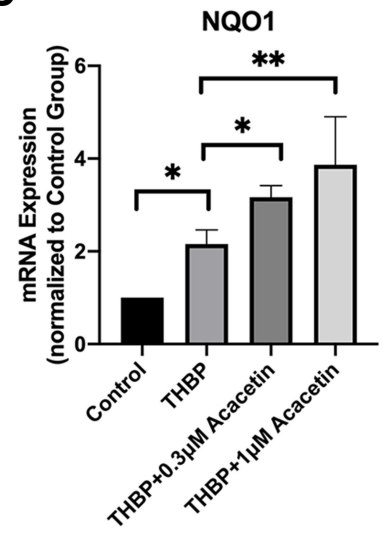

D

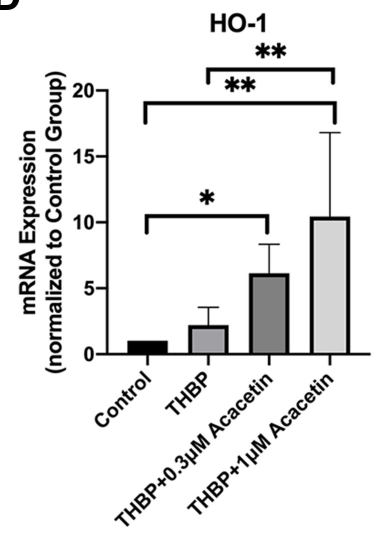

E

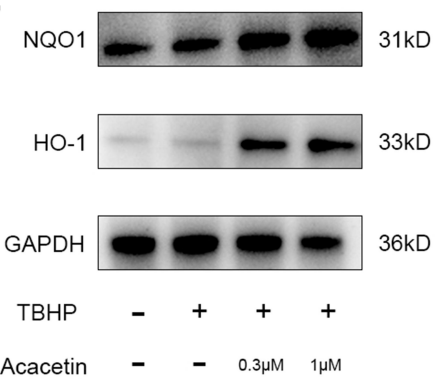

$\mathbf{F}$

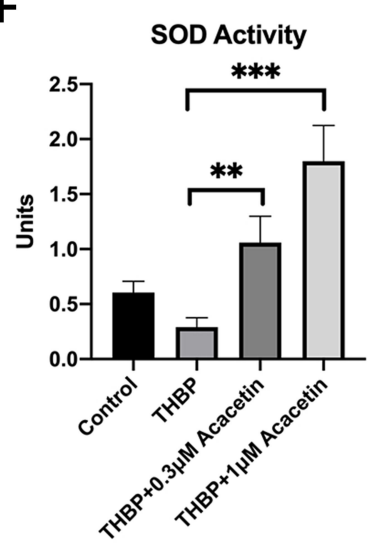

Figure 2 Acacetin suppressed TBHP-induced ROS generation and upregulated the expression and activity of antioxidant proteins. The NPCs were treated with TBHP (50 $\mu \mathrm{M})$ alone or in combination with acacetin $(0.3 \mu \mathrm{M}, \mathrm{I} \mu \mathrm{M})$ for 24 hours. (A, B) Intracellular ROS levels were determined using DCFH-DA with flow cytometry. (C, D) Relative mRNA expression levels of $\mathrm{HO}-\mathrm{I}$ and NQOI were determined by qPCR. (E) Protein expression levels of $\mathrm{HO}-\mathrm{I}$ and NQOI were determined by Western blotting. (F) SOD activities of the NPCs were determined using a SOD assay kit with WST-8. The data are represented as the mean \pm SD. $n=3$. ${ }^{2}<<0.05, * * p<0.01, * * * p<0.00 I$.

preincubated with acacetin for two hours and then treated with TBHP for another 24 hours. The proteins were extracted and analyzed by Western blotting. Figure 4B indicates that THBP activated the p38, JNK and ERK pathways through phosphorylation, while acacetin reversed their activation in a dose-dependent manner.

\section{Acacetin Alleviated Intervertebral Disc Degeneration in vivo}

To evaluate the effect of acacetin on IDD in vivo, we simulated IDD by puncturing the intervertebral discs of rats. After surgery, the rats were intraperitoneally injected with $25 \mathrm{mg} / \mathrm{kg}$ acacetin or an equal volume of saline once 
A

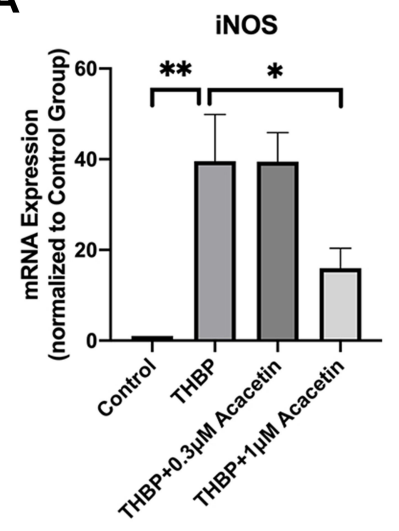

D

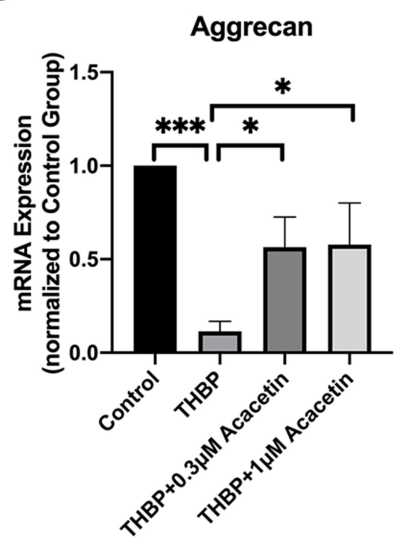

G

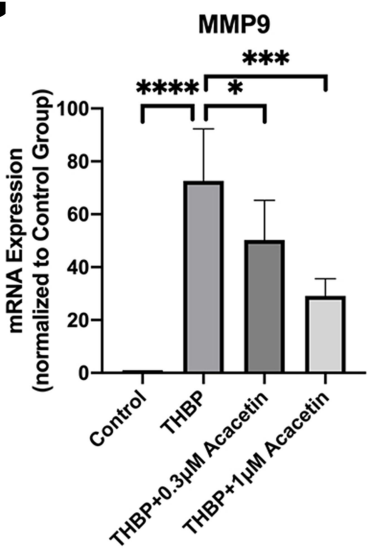

B

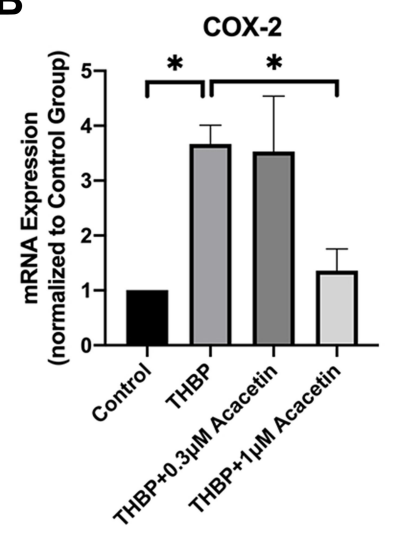

E

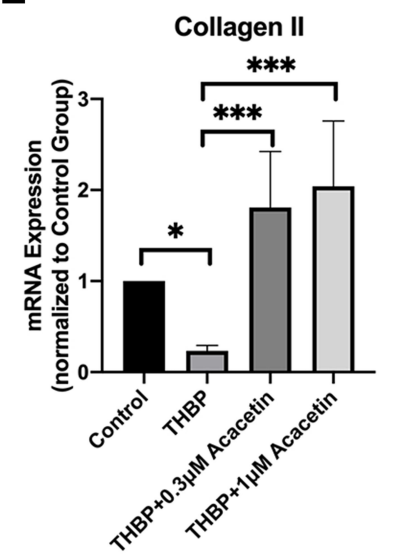

H

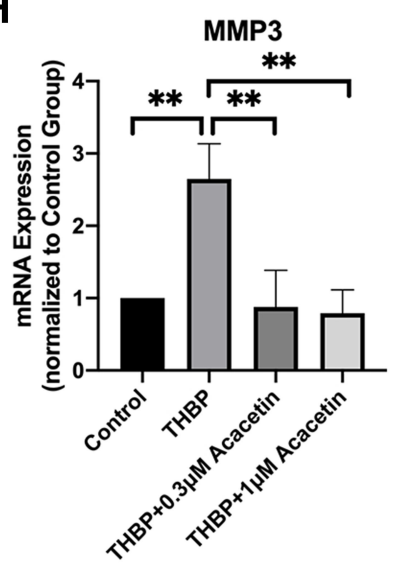

C

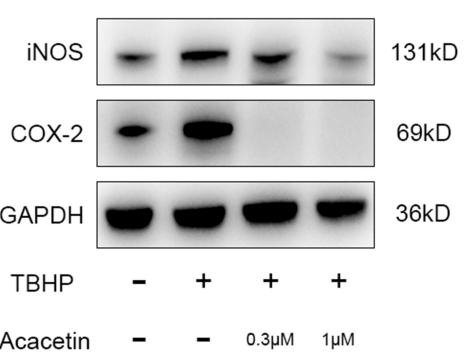

F

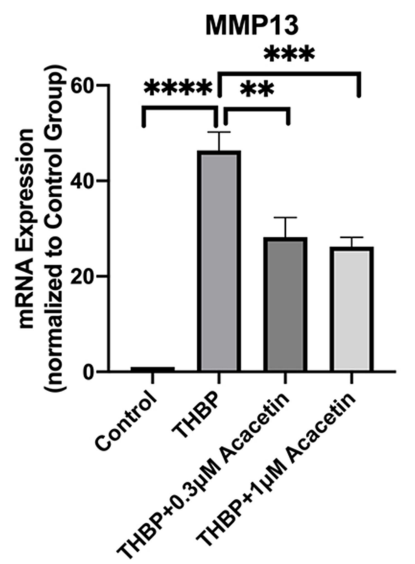

I

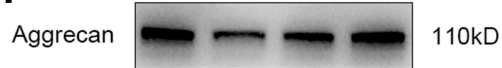

Collagen II $-\longrightarrow$ 142kD

MMP13 $=-\infty-\infty \mathrm{kD}$

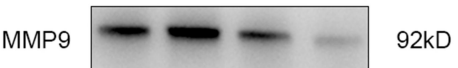

MMP3 $\quad 50 \mathrm{kD}$

GAPDH $36-\infty-\infty 6$

TBHP $\quad-\quad+\quad+\quad+$

Acacetin - $\quad-0.3 \mu \mathrm{M} 1 \mu \mathrm{M}$

Figure 3 Acacetin alleviated TBHP-induced inflammation and ECM degradation. The NPCs were treated with TBHP $(50 \mu M)$ alone or combination with acacetin $(0.3 \mu M$, I $\mu \mathrm{M}$ ) for 24 hours. (A, B) Relative mRNA expression levels of iNOS and COX-2 were determined by qPCR. (C) Protein expression levels of iNOS and COX-2 were evaluated using Western blotting. (D, E, F, G, H) Relative mRNA expression levels of aggrecan, collagen II, MMPI3, MMP9 and MMP3 were evaluated using qPCR. (I) Protein expression levels of aggrecan, collagen II, MMPI3, MMP9 and MMP3 were determined using Western blotting. Data are represented as the mean \pm SD. $n=3$. ${ }^{*}<0.05$, $* * p<0.01, * * * p<0.001, * * * * p<0.0001$.

a week. The degree of degeneration was evaluated using MRI on the day of surgery and four weeks later. Figure 5A shows that needle puncture did not immediately alter the
T2-weighted signal. Figure 5B shows that four weeks later, the T2-weighted signal decreased dramatically in the IDD group, whereas in the acacetin group, the signal 
A

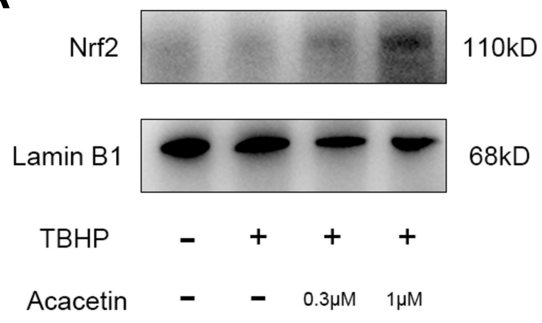

B

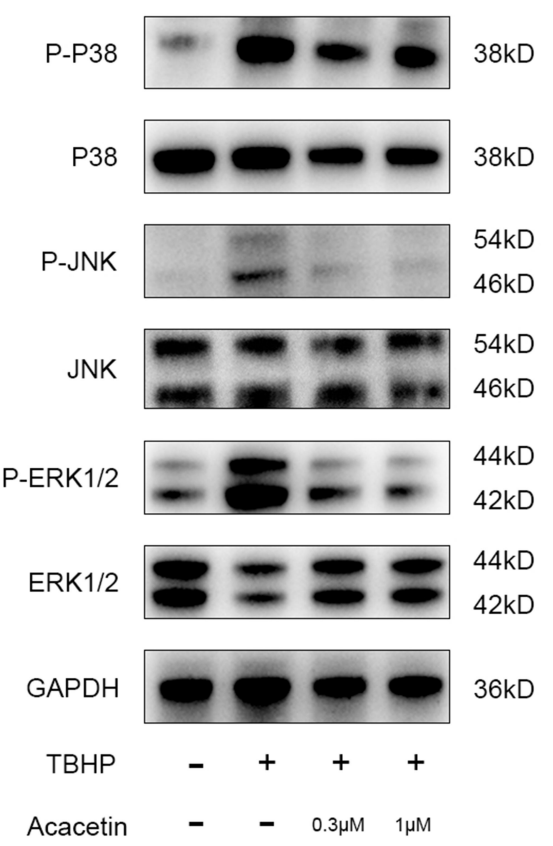

Figure 4 Acacetin activated the Nrf2 pathway and inhibited TBHP-induced activation of the MAPK pathway. (A) The NPCs were pretreated with acacetin (0.3 $\mu$ M, I $\mu$ M) for two hours and then treated with TBHP $(50 \mu \mathrm{M})$ for another 24 hours. The intranuclear protein content of Nrf2 was evaluated using Western blotting. (B) The NPCs were pretreated with acacetin $(0.3 \mu \mathrm{M}, \mathrm{I} \mu \mathrm{M})$ for two hours and then treated with TBHP $(50 \mu \mathrm{M})$ for another 24 hours. Protein expression levels of $\mathrm{p} 38$, P-p38, ERK, $\mathrm{p}$-ERK, JNK, and p-JNK were evaluated using Western blotting. The experiment was repeated three times.

\section{A}
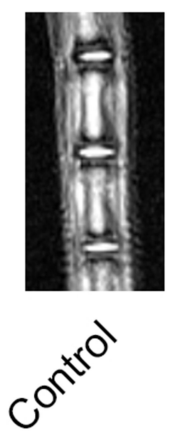

0 week
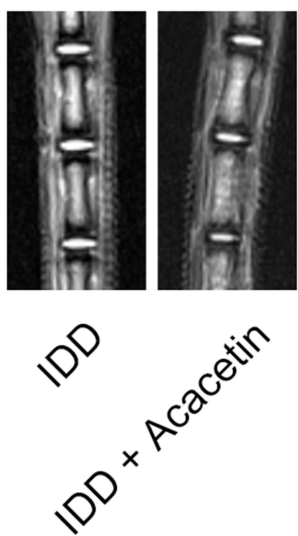

B
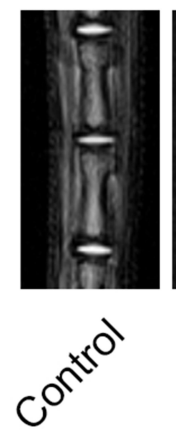

4 weeks
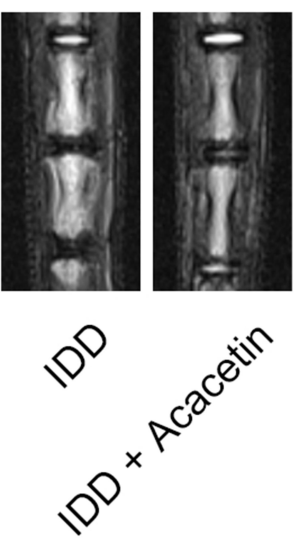

Figure 5 MRI evaluation of rat intervertebral disc. (A) T2-weighted MRI images of the rat tails immediately after the operation; (B) T2-weighted MRI images of the rat tails four weeks after the operation.

intensity was much higher than that in the IDD group but lower than that in the control group, indicating that acacetin could partially reverse the degeneration process.

As shown in Figure 6A, H\&E staining showed that the intervertebral discs of the control group were full of nucleus pulposus tissue and that the surrounding annulus fibrosus remained intact. Needle puncture obviously changed the structure of the disc. The gel-like nucleus pulposus was replaced by disorganized fibrocartilaginous tissue. The annulus fibrosus became invisible, and the distance between the vertebra was narrowed. The vertebra also exhibited bone destruction and hyperplasia. Acacetin application dramatically alleviated the observed disc degeneration. The nucleus pulposus remained partially intact, and the surrounding annulus fibrosus was slightly injured. Safranin O/fast green and $\mathrm{AB}$ staining demonstrated the proteoglycan and collagen 
A

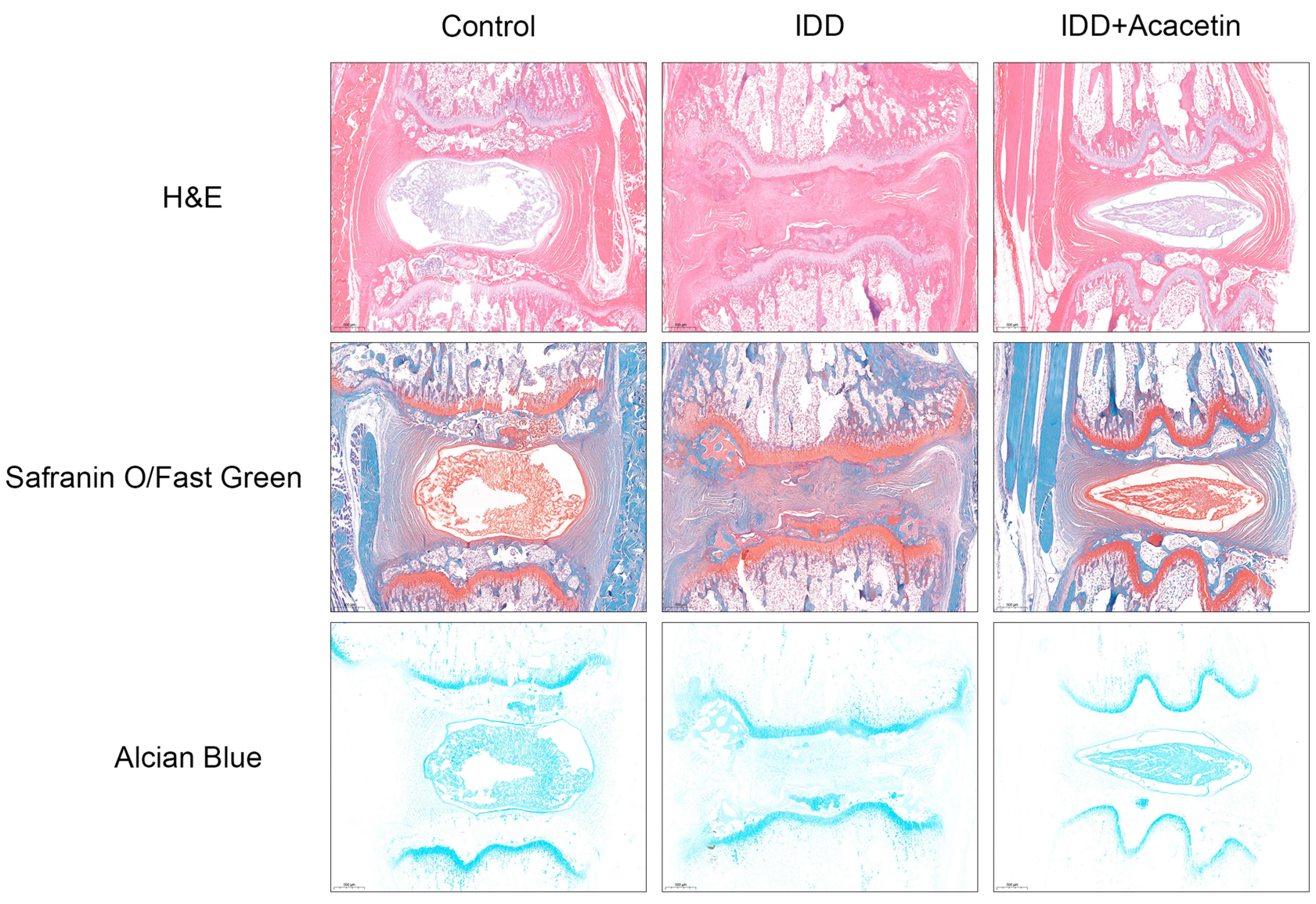

B

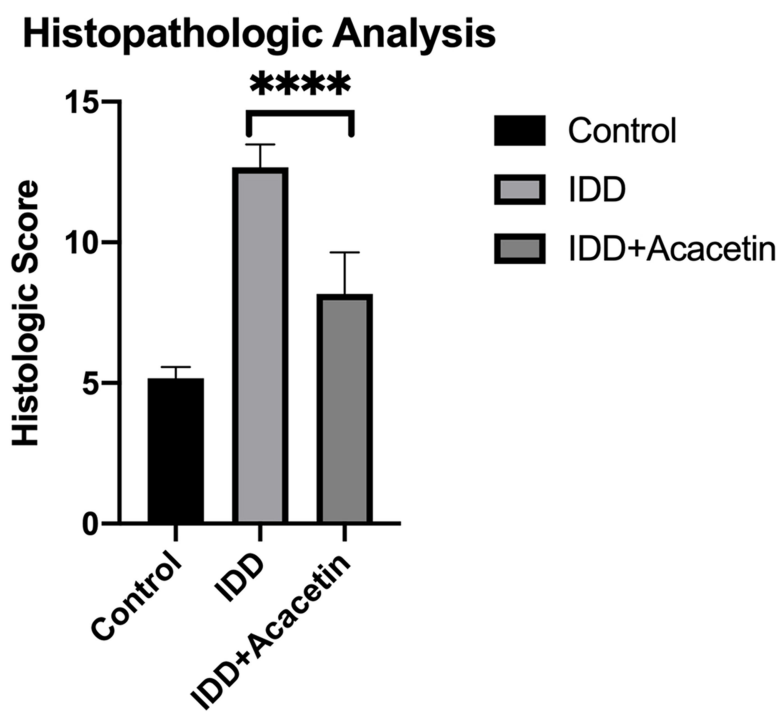

Figure 6 Histopathological analysis of the rat intervertebral disc. (A) H\&E, Safranin O/Fast Green and Alcian Blue staining of the rat intervertebral discs. (B) Histological score of the intervertebral discs. The data are represented as the mean $\pm S D$. $n=6$. ***** $p<0.0001$. 
contents. The color of the staining resulting from these two methods in the IDD group was much lighter than that in the control group, while the acacetin group exhibited relatively intense staining compared with that in the IDD group, indicating that acacetin partially reversed the observed disc degeneration. Finally, we used a histological score to compare the degree of degeneration in the different groups, and the results are shown in Figure 6B. The outcomes indicated that the acacetin group fared better than the IDD group.

\section{Discussion}

Low back pain is a leading contributor to the medical burden worldwide, and IDD is one of the major causes of low back pain. The common pharmacological therapies for IDD are NSAIDS and muscle relaxants, which can only relieve the symptoms. ${ }^{29}$ Therefore, there is an urgent need to find an effective and safe alternative to slow the progression of IDD. In this research, we demonstrated the therapeutic effect of acacetin on IDD. In vitro, acacetin alleviated the degradation of ECM and inhibited the generation of inflammatory mediators by scavenging intracellular ROS. The effect of this drug on IDD was found to be exerted by activating the Nrf2 pathway and blocking the ROS-induced activation of the MAPK pathway. In vivo, the intraperitoneal application of an appropriate dose of acacetin dramatically ameliorated puncture-induced IDD. This is the first study revealing the protective effect of acacetin on the nucleus pulposus. A schematic diagram of the mechanism is shown in Figure 7.

NPCs are responsible for the functional maintenance of the intervertebral discs. ROS generated during the degenerative process not only cause oxidative damage but also disrupt many signaling pathways that regulate redox reactions. $^{30}$ TBHP is a kind of organic peroxide that is more stable than ordinary hydrogen peroxide. Therefore, we treated NPCs with TBHP to simulate a high-ROS extracellular environment. Consistent with previous studies, our outcomes verified that TBHP led to inflammation and ECM degradation. ${ }^{31,32}$

Scavenging ROS can help relieve degeneration. Commonly used scavengers eliminate ROS through two mechanisms: reacting with ROS directly or enhancing the expression of intracellular antioxidant proteins. ${ }^{16}$ The former mechanism involves tempo, SOD, vitamin E and other components that can react with and eliminate ROS directly. $^{33,34}$ The latter mechanism involves many cytokines and natural or synthetic compounds. These molecules can regulate ROS-related signaling pathways and upregulate the expression of endogenous antioxidants. $^{35,36}$ Acacetin functions via the latter mechanism. Previous studies reported that acacetin is an activator of the Nrf2 pathway in other types of cells. ${ }^{25,27} \mathrm{Nrf2}$ is a key transcription factor existing in nearly all kinds of cells that regulates antioxidant processes. ${ }^{37} \mathrm{Nrf} 2$ binds with Kelch-like ECH-associated protein 1 (Keap1) in the cytoplasm and will be degraded through ubiquitination if inactivated. ${ }^{38}$ Nrf2 activity is controlled by Keap1 in a stress-dependent manner, and once Nrf2 is activated following various stimuli, it detaches from Keap1, translocates into the nucleus, and then binds to the antioxidant response element (ARE), initiating the synthesis of antioxidant proteins including NQO1, HO-1, SOD, glutamate cysteine ligase catalytic subunit (GCLC), and glutathioneS-transferases (GST). ${ }^{39-41}$ We treated NPCs with acacetin and analyzed the intranuclear content of the Nrf2 protein, demonstrating that acacetin also activated the Nrf2 pathway in NPCs. The downstream protein expression levels of NQO1, HO-1 and SOD were also dramatically upregulated.

Existing studies have shown that excessive ROS disrupt the MAPK pathway. ${ }^{42}$ The MAPK pathway consists of a series of proteins regulating growth, differentiation and inflammation. p38, JNK and ERK are the major components of the MAPK pathway. These proteins are activated through phosphorylation. ${ }^{43}$ ROS activate $\mathrm{p} 38$, JNK and ERK and continuously upregulate the expression of downstream MMPs, ADAMTS (a disintegrin and metalloproteinase with thrombospondin motifs) and inflammatory mediators such as NO and PGE2, ultimately resulting in ECM degradation and inflammation. ${ }^{44}$ Our research demonstrated that acacetin inhibited the phosphorylation of p38, JNK and ERK1/2. However, it remains unknown whether acacetin inhibits phosphorylation directly or by reducing ROS generation.

This research has several limitations. First, IDD is a complicated physiological process. TBHP application cannot completely mimic the environment of NPCs. Multiple inflammatory mediators, such as IL-1 $\beta$ and TNF- $\alpha$, participate in the degeneration process, and these mediators have also been used to simulate the degenerative environment. ${ }^{45,46}$ We used TBHP to simulate the degeneration process because this study was focused on ROS, and TBHP is normally used for highROS environment simulation. However, the coadministration of TBHP and other inflammatory mediators might better mimic the degenerative environment. Second, in 


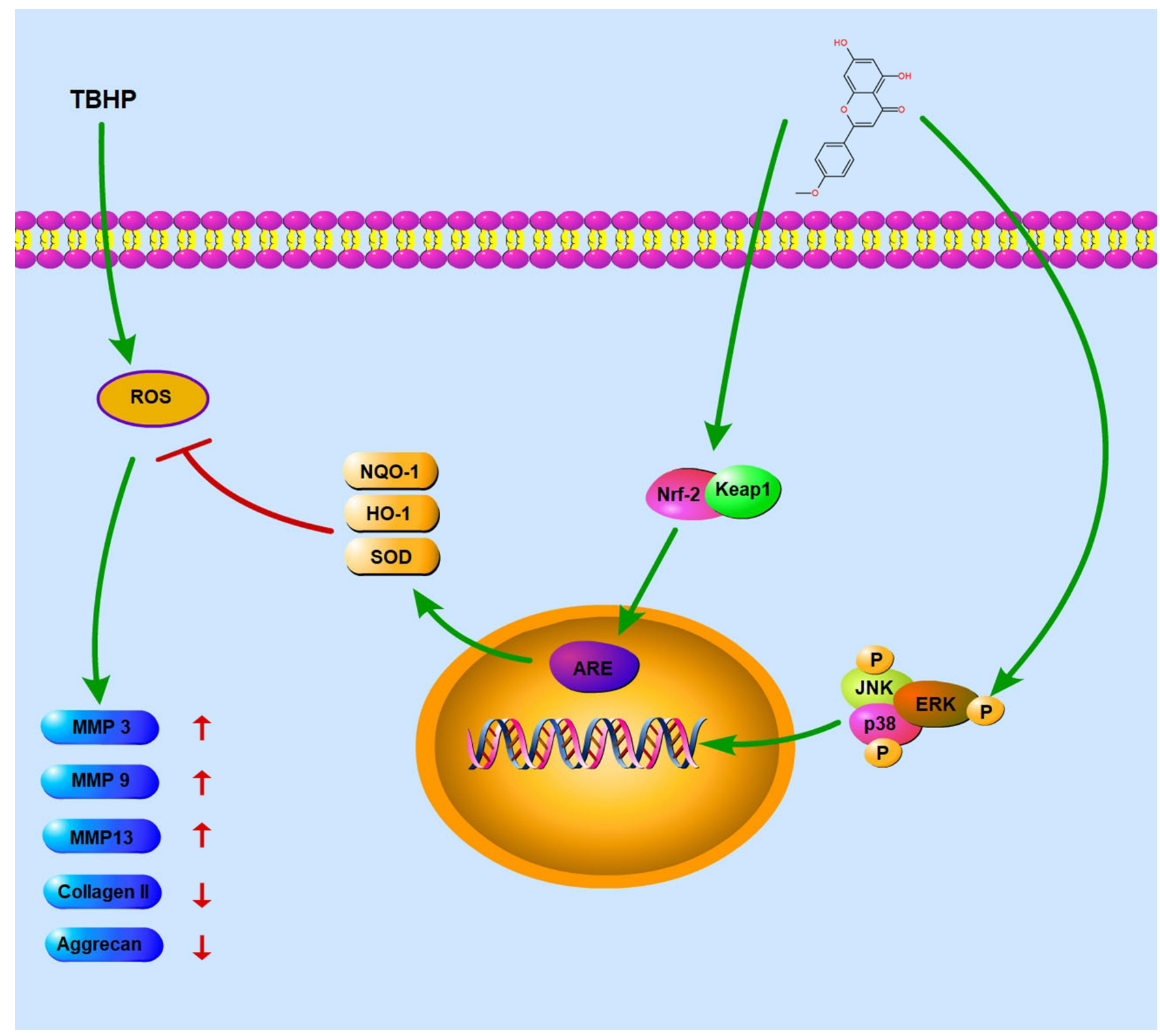

Figure 7 Schematic diagram of the mechanism of action. TBHP facilitates the generation of ROS, which upregulates the expression of inflammatory mediators and accelerates the degradation of ECM. Acacetin scavenges ROS through the Nrf2 pathway and inhibits the p38, JNK, ERK pathways to relieve the degenerative process.

our in vivo study, acacetin was administered intraperitoneally. This drug may migrate to the intervertebral disc and protect NPCs directly. However, it is also possible that acacetin affects other physiological processes and protects intervertebral discs indirectly. The detailed mechanism of action in vivo may be further elucidated by labeling the drug and tracing its distribution and metabolism. In addition, the crosstalk between the Nrf2 pathway and the MAPK pathway has not been studied in this research. A number of studies have indicated that the activation of JNK1 and ERK2 promotes the activation of the Nrf2 pathway, while the activation of the p38 pathway has an inhibitory effect. ${ }^{47}$ The detailed interactions between these two signaling pathways in NPCs require further elucidation. Finally, the safety of this drug in rats or larger animals needs to be evaluated before clinical trials.

\section{Conclusion}

In summary, our research showed that acacetin alleviated TBHP-induced ROS generation, inflammation and ECM degradation by activating the Nrf2 pathway and suppressing the MAPK pathway. In addition, acacetin ameliorated the degenerative process in a rat disc puncture model in vivo. These outcomes indicated that acacetin may be a potential treatment for IDD. 


\section{Abbreviations}

IDD, intervertebral disc degeneration; NPC, nucleus pulposus cell; ROS, reactive oxygen species; TBHP, tert-butyl peroxide; ECM, extracellular matrix; IL-1 $\beta$, interleukin-1 $\beta$; TNF- $\alpha$, tumor necrosis factor- $\alpha$; NO, nitric oxide; PGE2, prostaglandin E2; NF-кB, nuclear factor kappa-B; MAPK, mitogenactivated protein kinase; PVDF, polyvinylidene difluoride; CCK-8, Cell Counting Kit-8; SDS-PAGE, sodium dodecyl sulfate-polyacrylamide gel electrophoresis; SOD, superoxide dismutase; HO-1, heme oxygenase-1; NQO1, NAD(P)H dehydrogenase, quinone 1; MMP, matrix metalloproteinase; JNK, c-JUN N-terminal kinase; ERK, extracellular signalregulated kinase; Keap1, Kelch-like ECH-associated protein 1; ARE, antioxidant response element; iNOS, inducible nitric oxide synthase; COX-2, cyclooxygenase 2 .

\section{Acknowledgments}

We are grateful for the support from the central laboratory of Zhongshan Hospital.

\section{Disclosure}

The authors report no conflicts of interest in this work.

\section{References}

1. Dudek M, Yang N, Ruckshanthi JP, et al. The intervertebral disc contains intrinsic circadian clocks that are regulated by age and cytokines and linked to degeneration. Int J Mol Sci. 2017;76(3):576-584.

2. Yu H, Liu Y, Xie W, Xie Q, Liu Q, Cheng L. IL-38 alleviates the inflammatory response and the degeneration of nucleus pulposus cells via inhibition of the NF- $\mathrm{BB}$ signaling pathway in vitro. Int Immunopharmacol. 2020;85:106592. doi:10.1016/j. intimp.2020.106592

3. Cazzanelli P, Wuertz-Kozak K. MicroRNAs in Intervertebral Disc Degeneration, Apoptosis, Inflammation, and Mechanobiology. Int J Mol Sci. 2020;21(10):10. doi:10.3390/ijms21103601

4. Swank KR, Furness JE, Baker EA, Gehrke CK, Biebelhausen SP, Baker KC. Metabolomic Profiling in the Characterization of Degenerative Bone and Joint Diseases. Metabolites. 2020;10(6):6. doi:10.3390/metabo10060223

5. Clouet J, Vinatier C, Merceron C, et al. The intervertebral disc: from pathophysiology to tissue engineering. Joint Bone Spine. 2009;76 (6):614-618. doi:10.1016/j.jbspin.2009.07.002

6. Yang S, Zhang F, Ma J, Ding W. Intervertebral disc ageing and degeneration: the antiapoptotic effect of oestrogen. Ageing Res Rev. 2020;57:100978. doi:10.1016/j.arr.2019.100978

7. Risbud MV, Shapiro IM. Role of cytokines in intervertebral disc degeneration: pain and disc content. Nat Rev Rheumatol. 2014;10 (1):44-56. doi:10.1038/nrrheum.2013.160

8. Lin J, Chen J, Zhang Z, et al. Luteoloside Inhibits IL-1 $\beta$-Induced Apoptosis and Catabolism in Nucleus Pulposus Cells and Ameliorates Intervertebral Disk Degeneration. Front Pharmacol. 2019; 10:868.

9. Liu Y, Li Y, Nan L-P, et al. Insights of stem cell-based endogenous repair of intervertebral disc degeneration. World J Stem Cells. 2020;12 (4):266-276. doi:10.4252/wjsc.v12.i4.266
10. Cunha C, Q. Teixeira G, Ribeiro-Machado C. Modulation of the In Vivo Inflammatory Response by Pro- Versus Anti-Inflammatory Intervertebral Disc Treatments. Int $J$ Mol Sci. 2020;21(5):5. doi:10.3390/ijms21051730

11. Lin D, Alberton P, Delgado Caceres M, et al. Loss of tenomodulin expression is a risk factor for age-related intervertebral disc degeneration. Aging Cell. 2020;19(3):e13091. doi:10.1111/acel.13091

12. Bai Z, Liu W, He D, et al. Protective effects of autophagy and NFE2L2 on reactive oxygen species-induced pyroptosis of human nucleus pulposus cells. Aging. 2020;12(8):7534-7548. doi:10.18632/ aging. 103109

13. Fang C, Gu L, Smerin D, Mao S, Xiong X. The Interrelation between Reactive Oxygen Species and Autophagy in Neurological Disorders. Oxid Med Cell Longev. 2017;2017:8495160. doi:10.1155/2017/8495160

14. Ge J, Zhou Q, Cheng X, et al. The protein tyrosine kinase inhibitor, Genistein, delays intervertebral disc degeneration in rats by inhibiting the p38 pathway-mediated inflammatory response. Aging. 2020;12 (3):2246-2260. doi:10.18632/aging.102743

15. Chen F, Liu H, Wang X, et al. Melatonin activates autophagy via the $\mathrm{NF}-\kappa \mathrm{B}$ signaling pathway to prevent extracellular matrix degeneration in intervertebral disc. Osteoarthritis Cartilage. 2020;28 (8):1121-1132. doi:10.1016/j.joca.2020.05.011

16. Feng C, Yang M, Lan M, et al. ROS: crucial Intermediators in the Pathogenesis of Intervertebral Disc Degeneration. Oxid Med Cell Longev. 2017;2017:5601593. doi:10.1155/2017/5601593

17. Kwon EB, Kang MJ, Ryu HW, et al. Acacetin enhances glucose uptake through insulin-independent GLUT4 translocation in L6 myotubes. Phytomedicine. 2020;68:153178. doi:10.1016/j.phymed.2020.153178

18. Xiao WZ, Zhou WH, Ma Q, Cui WG, Mei QY, Zhao X. Serotonergically dependent antidepressant-like activity on behavior and stress axis responsivity of acacetin. Pharmacol Res. 2019;146:104310. doi:10.1016/j.phrs.2019.104310

19. Sun F, Li D, Wang C, Peng C, Zheng H, Wang X. Acacetin-induced cell apoptosis in head and neck squamous cell carcinoma cells: evidence for the role of muscarinic M3 receptor. Phytother Res. 2019;33(5):1551-1561. doi:10.1002/ptr.6343

20. Kim SM, Park YJ, Shin MS, et al. Acacetin inhibits neuronal cell death induced by 6-hydroxydopamine in cellular Parkinson's disease model. Bioorg Med Chem Lett. 2017;27(23):5207-5212. doi:10.1016/ j.bmcl.2017.10.048

21. Bu J, Shi S, Wang HQ, et al. Acacetin protects against cerebral ischemia-reperfusion injury via the NLRP3 signaling pathway. Neural Regeneration Res. 2019;14(4):605-612. doi:10.4103/1673-5374.247465

22. Liu H, Kang H, Song C, et al. Urolithin A Inhibits the Catabolic Effect of TNF $\alpha$ on Nucleus Pulposus Cell and Alleviates Intervertebral Disc Degeneration in vivo. Front Pharmacol. 2018;9:1043. doi:10.3389/fphar.2018.01043

23. Mao HJ, Chen QX, Han B, et al. The effect of injection volume on disc degeneration in a rat tail model. Spine. 2011;36(16):E10621069. doi:10.1097/BRS.0b013e3182027d42

24. Feng P, Ding H, Lin H, Chen W. AOD: the antioxidant protein database. Sci Rep. 2017;7(1):7449. doi:10.1038/s41598-017-08115-6

25. Wu WY, Li YD, Cui YK, et al. The Natural Flavone Acacetin Confers Cardiomyocyte Protection Against Hypoxia/Reoxygenation Injury via AMPK-Mediated Activation of Nrf2 Signaling Pathway. Front Pharmacol. 2018;9:497. doi:10.3389/fphar.2018.00497

26. Shang P, Tang Q, Hu Z, et al. Procyanidin B3 alleviates intervertebral disc degeneration via interaction with the TLR4/MD-2 complex. J Cell Mol Med. 2020;24(6):3701-3711. doi:10.1111/jcmm.15074

27. Wu D, Wang Y, Zhang H, Du M, Li T. Acacetin attenuates mice endotoxin-induced acute lung injury via augmentation of heme oxygenase-1 activity. Inflammopharmacology. 2018;26(2):635-643.

28. Jin $\mathrm{H}$, Wang $\mathrm{Q}, \mathrm{Wu} \mathrm{J}$, et al. Baicalein Inhibits the IL-1 $\beta$-Induced Inflammatory Response in Nucleus Pulposus Cells and Attenuates Disc Degeneration In vivo. Inflammation. 2019;42(3):1032-1044. doi:10.1007/s10753-019-00965-8 
29. Maher C, Underwood M, Buchbinder R. Non-specific low back pain. Lancet. 2017;389(10070):736-747. doi:10.1016/S0140-6736(16) 30970-9

30. Xie L, Huang W, Fang Z, et al. CircERCC2 ameliorated intervertebral disc degeneration by regulating mitophagy and apoptosis through miR-182-5p/SIRT1 axis. Cell Death Dis. 2019;10(10):751. doi:10.1038/s41419-019-1978-2

31. Liu X, Jiang C, Liu G, et al. Sodium butyrate protects against oxidative stress in human nucleus pulposus cells via elevating PPAR $\gamma$-regulated Klotho expression. Int Immunopharmacol. 2020;85:106657. doi:10.1016/j.intimp.2020.106657

32. $\mathrm{Xu} \mathrm{WN}$, Zheng HL, Yang RZ, et al. Mitochondrial NDUFA4L2 attenuates the apoptosis of nucleus pulposus cells induced by oxidative stress via the inhibition of mitophagy. Exp Mol Med. 2019;51 (11):1-16.

33. Kavanaugh TE, Dailing EA, Cho H, Hasty KA, Duvall CL. Development of Optimized Copolymers and Delivery Formulations to Scavenge Reactive Oxygen Species and Prevent Joint Damage from Post-Traumatic Osteoarthritis. Osteoarthritis Cartilage. 2017;25:S265-S266. doi:10.1016/j.joca.2017.02.447

34. Zeng Z, Han N, Liu C, et al. Functional dissection of HGGT and HPT in barley vitamin E biosynthesis via CRISPR/Cas9-enabled genome editing. Ann Bot. 2020;126(5):929-942. doi:10.1093/aob/mcaa115

35. Yao X, Zhang J, Jing $\mathrm{X}$, et al. Fibroblast growth factor 18 exerts anti-osteoarthritic effects through PI3K-AKT signaling and mitochondrial fusion and fission. Pharmacol Res. 2019;139:314-324. doi:10.1016/j.phrs.2018.09.026

36. Chen ZB, Yu YB, Wa QB, Zhou JW, He M, Cen Y. The role of quinazoline in ameliorating intervertebral disc degeneration by inhibiting oxidative stress and anti-inflammation via NF- $\mathrm{B} / \mathrm{MAPKs}$ signaling pathway. Eur Rev Med Pharmacol Sci. 2020;24 (4):2077-2086.

37. Moi P, Chan K, Asunis I, Cao A, Kan YW. Isolation of NF-E2-related factor 2 (Nrf2), a NF-E2-like basic leucine zipper transcriptional activator that binds to the tandem NF-E2/AP1 repeat of the beta-globin locus control region. Proc Natl Acad Sci U S A. 1994;91(21):9926-9930. doi:10.1073/pnas.91.21.9926

38. Sharma V, Kaur A, Singh TG. Counteracting role of nuclear factor erythroid 2-related factor 2 pathway in Alzheimer's disease. Biomed Pharma. 2020;129:110373. doi:10.1016/j.biopha.2020.110373
39. Hayashi M, Kuga A, Suzuki M, et al. Microenvironmental activation of Nrf2 restricts the progression of Nrf2-activated malignant tumors. Cancer Res. 2020;80(16):3331-3344. doi:10.1158/0008-5472.CAN19-2888

40. Yang HL, Yang TY, Gowrisankar YV, et al. Suppression of LPSInduced Inflammation by Chalcone Flavokawain A through Activation of Nrf2/ARE-Mediated Antioxidant Genes and Inhibition of ROS/NFאB Signaling Pathways in Primary Splenocytes. Oxid Med Cell Longev. 2020;2020:3476212. doi:10.1155/2020/3476212

41. Mohsin Alvi A, Tariq Al Kury L, Umar Ijaz M. Tariq Al Kury L, Umar Ijaz M, et al. Post-Treatment of Synthetic Polyphenolic 1,3,4 Oxadiazole Compound A3, Attenuated Ischemic Stroke-Induced Neuroinflammation and Neurodegeneration. Biomolecules. 2020;10 (6):6. doi:10.3390/biom 10060816

42. Zhang Z, Nian Q, Chen G, Cui S, Han Y, Zhang J. Klotho Alleviates Lung Injury Caused by Paraquat via Suppressing ROS/P38 MAPK-Regulated Inflammatory Responses and Apoptosis. Oxid Med Cell Longev. 2020;2020:1854206.

43. Chen H, Fang C, Zhi X, et al. Neobavaisoflavone inhibits osteoclastogenesis through blocking RANKL signalling-mediated TRAF6 and

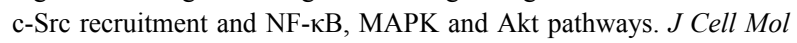
Med. 2020;2145.

44. Wu Y, Wang Z, Fu X, Lin Z, Yu K. Geraniol-mediated osteoarthritis improvement by down-regulating PI3K/Akt/NF- $\mathrm{BB}$ and MAPK signals: in vivo and in vitro studies. Int Immunopharmacol. 2020;86:106713. doi:10.1016/j.intimp.2020.106713

45. Yuan Q, Wang X, Liu L, et al. Exosomes derived from human placental Mesenchymal Stromal Cells carrying miR-4450 inhibitor alleviate intervertebral disc degeneration and ameliorate gait disturbances via up-regulation of ZNF121. Stem Cells Dev. 2020;29 (16):1038-1058. doi:10.1089/scd.2020.0083

46. Zou L, Lei H, Shen J, et al. HO-1 induced autophagy protects against IL-1 $\beta$-mediated apoptosis in human nucleus pulposus cells by inhibiting NF-кB. Aging. 2020;12(3):2440-2452. doi:10.18632/ aging. 102753

47. Huang Y, Li W, Su ZY, Kong AN. The complexity of the Nrf2 pathway: beyond the antioxidant response. J Nutr Biochem. 2015;26(12):1401-1413. doi:10.1016/j.jnutbio.2015.08.001
Drug Design, Development and Therapy

\section{Publish your work in this journal}

Drug Design, Development and Therapy is an international, peerreviewed open-access journal that spans the spectrum of drug design and development through to clinical applications. Clinical outcomes, patient safety, and programs for the development and effective, safe, and sustained use of medicines are a feature of the journal, which has also been accepted for indexing on PubMed Central. The manuscript management system is completely online and includes a very quick and fair peer-review system, which is all easy to use. Visit http://www. dovepress.com/testimonials.php to read real quotes from published authors. 